DRAFT VERSION MAY 2, 2018

Preprint typeset using LATEX style emulateapj v. 5/2/11

\title{
HELIUM IN NATAL H II REGIONS: THE ORIGIN OF THE X-RAY ABSORPTION IN GAMMA-RAY BURST AFTERGLOWS
}

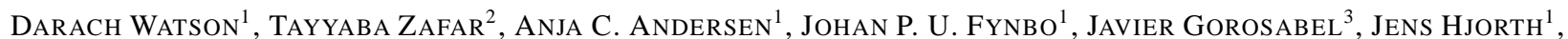 \\ PÁll Jakobsson $^{4}$, Thomas KrÜhler ${ }^{1}$, Peter Laursen ${ }^{1}$, Giorgos Leloudas ${ }^{1,5}$, ANd Daniele Malesani ${ }^{1}$ \\ Draft version May 2, 2018
}

\begin{abstract}
Soft X-ray absorption in excess of Galactic is observed in the afterglows of most gamma-ray bursts (GRBs), but the correct solution to its origin has not been arrived at after more than a decade of work, preventing its use as a powerful diagnostic tool. We resolve this long-standing problem and find that absorption by $\mathrm{He}$ in the GRB's host H II region is responsible for most of the absorption. We show that the X-ray absorbing column density $\left(N_{\mathrm{H}_{\mathrm{X}}}\right)$ is correlated with both the neutral gas column density and with the optical afterglow's dust extinction $\left(A_{V}\right)$. This correlation explains the connection between dark bursts and bursts with high $N_{\mathrm{H}_{\mathrm{X}}}$ values. From these correlations we exclude an origin of the X-ray absorption which is not related to the host galaxy, i.e. the intergalactic medium or intervening absorbers are not responsible. We find that the correlation with the dust column has a strong redshift evolution, whereas the correlation with the neutral gas does not. From this we conclude that the column density of the X-ray absorption is correlated with the total gas column density in the host galaxy rather than the metal column density, in spite of the fact that X-ray absorption is typically dominated by metals. The strong redshift evolution of $N_{\mathrm{H}_{\mathrm{X}}} / A_{V}$ is thus a reflection of the cosmic metallicity evolution of star-forming galaxies and we find it to be consistent with measurements of the redshift evolution of metallicities for GRB host galaxies. We conclude that the absorption of X-rays in GRB afterglows is caused by $\mathrm{He}$ in the $\mathrm{H}$ II region hosting the GRB. While dust is destroyed and metals are stripped of all of their electrons by the GRB to great distances, the abundance of He saturates the He-ionising UV continuum much closer to the GRB, allowing it to remain in the neutral or singly-ionised state. Helium X-ray absorption explains the correlation with total gas, the lack of strong evolution with redshift as well as the absence of dust, metal or hydrogen absorption features in the optical-UV spectra.
\end{abstract}

Subject headings: gamma-ray burst: general — early universe — dark ages, reionization, first stars — galaxies: ISM

\section{INTRODUCTION}

Long-duration gamma-ray bursts (GRBs) accompany the deaths of some stripped-envelope massive stars (Hjorth et al. 2003; Stanek et al. 2003). And while they have been acknowledged as excellent probes of both the typical interstellar medium of their host galaxies and foreground absorbers (e.g. Jakobsson et al. 2004), their use as probes of the progenitor star's circumstellar material has met with limited success. The main difficulty in using GRBs to examine their immediate environs is that they are so luminous across all wavelengths that they can be expected to ionise gas and destroy dust to very large distances from the burst (Waxman \& Draine 2000; Fruchter et al. 2001; Perna et al. 2003), potentially eradicating all traces of the circumstellar environment from their spectra, except potentially in hot gas. The best hope for observing the environment near the burst could therefore be at X-ray wavelengths.

From the early soft X-ray observations of GRBs, large

\footnotetext{
${ }^{1}$ Dark Cosmology Centre, Niels Bohr Institute, University of Copenhagen, Juliane Maries Vej 30, DK-2100 Copenhagen Ø, Denmark; darachedark-cosmology.dk

${ }^{2}$ Laboratoire d'Astrophysique de Marseille - LAM, Université AixMarseille \& CNRS, UMR 7326, 38 rue F. Joliot-Curie, 13388, Marseille Cedex 13, France

${ }^{3}$ Instituto de Astrofísica de Andalucía (IAA-CSIC), Glorieta de la Astronomía s/n, 18008, Granada, Spain

${ }^{4}$ Centre for Astrophysics and Cosmology, Science Institute, University of Iceland, Dunhagi 5, 107, Reykjavik, Iceland

${ }^{5}$ The Oskar Klein Centre, Department of Physics, Stockholm University, 10691, Stockholm, Sweden
}

absorptions were observed, initially believed to be consistent with the light from the afterglow making its way out of a molecular cloud and suffering absorption from the metals in the molecular cloud (Galama \& Wijers 2001; Reichart \& Price 2002; Campana et al. 2006a). However, it has become clear since then that the picture is not that simple. Indeed, the distribution of X-ray absorbing column densities is substantially larger than one would expect in such a scenario. And it has been shown that the X-ray absorption is not readily connected to either the neutral hydrogen column observed in the optical whether corrected for metallicity or not, to low-ionisation gas, or to the column of dust (Watson et al. 2007; Campana et al. 2010; Schady et al. 2011; Zafar et al. 2011).

As we progress in our understanding, the nature of the Xray downturn at soft $\mathrm{X}$-rays seems to become less and less clear. We still do not know how it arises, and indeed we are beginning to question whether it is even due to photoelectric absorption (Butler \& Kocevski|2007). This question has been a decade in the making (Galama \& Wijers 2001; Watson et al. 2002) and is one of the outstanding observational issues related to GRBs. Many suggestions have been made as to its origin, among them highly-ionised gas in the intergalactic medium (IGM), the host galaxy molecular cloud, or circumstellar material related to the GRB progenitor.

If we can resolve the origin of the X-ray absorption, we may find out about the immediate environment of the GRB, and hence know about its progenitor and the matrix in which it was created; or, if recent suggestions are correct (Behar et al. 2011), resolve the missing baryons problem by detecting the 
warm-hot intergalactic medium.

In $\S 2$ we present the problematic interpretation of the existing data and discuss how the X-ray column density is determined and presented. In $\S 3$ we present our results on the correspondence between the X-ray absorbing column density and the gas and dust columns. The origin of the X-ray absorption is explored in $\S 4$, while its specific properties are discussed in $\S 5$. The implications of our findings are examined in $\$ 6$ and our conclusions presented in $\S 7$.

\section{OBSERVATIONAL FACTS ABOUT X-RAY ABSORBERS IN GRBS}

We now array the facts we know about the X-ray absorption in GRB afterglows.

In the Galaxy, there are strong correlations between the neutral hydrogen column density, $N_{\mathrm{H}}$, the dust column density, $A_{V}$, and the X-ray absorbing column density, $N_{\mathrm{Hx}}$ (Burstein \& Heiles 1978; Bohlin et al. 1978; Shull \& van Steenberg 1985; Diplas \& Savage 1994; Schlegel et al. 1998; Gorenstein 1975; Predehl \& Schmitt 1995; Rachford et al. 2009; Güver \& Özel 2009; Watson 2011). The proportionality constant is different in the Magellanic Clouds for $N_{\mathrm{H}} / N_{\mathrm{H}_{\mathrm{X}}}$, but corrected for the lower metallicities of these galaxies, the ratio is quite similar (Koornneef 1982; Bouchet et al. 1985; Fitzpatrick 1985ab, 1986; Martin et al. 1989; Gordon et al. 2003; Dobashi et al. 2008; Welty et al. 2012). The metals-to-dust ratio, $N_{\mathrm{H}_{\mathrm{X}}} / A_{V}$, is relatively constant not only in local group galaxies (Welty et al. 2012), but also in galaxies at cosmological redshifts (e.g. Dai et al. 2006; Dai \& Kochanek 2009). However in GRB afterglows, the comparison with $\mathrm{H}$ I and $A_{V}$ shows that $N_{\mathrm{H}_{\mathrm{X}}}$ is significantly larger than expected, whether metallicity corrections are included or not (Zafar et al. 2011; Campana et al. 2012). $\quad N_{\mathrm{H}_{\mathrm{X}}} / A_{V}$ is typically an order of magnitude larger in GRBs than observed in other galaxies with a wide variation (Watson et al. 2006, 2007; Schady et al. 2010; Zafar et al. 2011). Finally, it was recently shown that the $N_{\mathrm{Hx}} / A_{V}$ ratio evolves strongly with redshift (Watson \& Jakobsson 2012). This is strange and so far remains unexplained. We explore this phenomenon below.

The consensus view has so far been that the bulk of X-ray absorption is not due to what is causing the dust extinction and gas absorption observed in the optical and is probably therefore due to very highly ionised gas (Watson et al. 2007; Campana et al. 2010; Schady et al. 2011; Campana et al. 2012).

\subsection{Practical considerations}

The downturn observed in the soft X-ray spectra of GRB afterglows (Galama \& Wijers 2001; Watson et al. 2002) was initially assumed to be the same as that observed in the Galaxy and in most extragalactic sources: photoelectric absorption due to metals along the line of sight. This absorption is primarily due to the inner shell electrons of the most abundant metals. In the energy range observed by most modern X-ray detectors (approximately $0.2-10 \mathrm{keV}$ ) these metals are in particular $\mathrm{C}, \mathrm{N}, \mathrm{O}, \mathrm{Ne}$, and $\mathrm{S}$ with contributions from the L-shell of $\mathrm{Fe}$ (see for example Wilms et al. 2000) and a contribution from He. Oxygen provides approximately $40 \%$ of the opacity in the Swift-XRT's passband at $z=0$. Because the absorption is caused by the inner shell electrons, mildly ionised gas still absorbs soft X-rays, as do atoms in dust or molecules, with relatively little alteration. Thus, soft X-ray absorption is a means to determine the total column density in metals in front

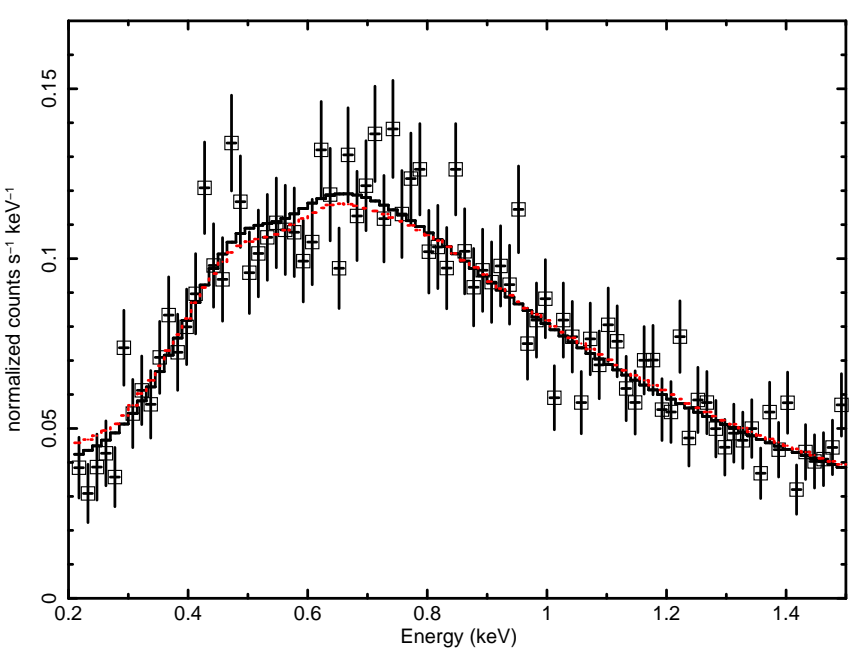

Figure 1. Soft X-ray spectrum of the afterglow of GRB 121027A, convolved with the XMM-Newton instrument response. The best-fit models for a powerlaw with a neutral, solar metallicity absorber (black, solid line), and a pure He absorber (red, dashed line) both at $z=1.773$ are shown. The models are practically indistinguishable.

of an object. Unfortunately, the low effective spectral resolution to absorption features of all of our soft X-ray spectra is such that individual features cannot be distinguished (including the spectra obtained with XMM-Newton and Chandra's gratings, since the total numbers of counts obtained with these instruments is so low, e.g. Mirabal et al. 2003; Butler et al. 2003, 2005; Campana et al. 2011).

It's worth noting here that at low metallicities, the absorption is increasingly dominated by $\mathrm{He}$ (and to a lesser extent, $\mathrm{H}$, if it exists in the neutral phase), and there is no clear way to distinguish absorption by different elements. As an illustration, in Fig. 1, we show the spectrum of the X-ray afterglow of GRB 121027A at $z=1.773$ (Tanvir et al. 2012; Krühler et al. 2012a), fitted with a traditional solar abundance absorber, and with a pure He absorber. The difference between the fits for the models is negligible. However, the total column density in $\mathrm{He}$ is nearly a factor of seven larger for the pure $\mathrm{He}$ absorber. For singly-ionised He, this is another factor of $\sim 25 \%$ larger again, since the cross-section for neutral He is somewhat larger in soft X-rays than for $\mathrm{He}^{+}$. This means that for a $\mathrm{H}$ II region, with a considerable fraction of He II but no metals, the gas column density would be about 8 times the column required for a neutral, solar metallicity gas at this redshift.

The lack of spectral resolution good enough to discriminate individual features gives rise to complications and confusion in the presentation of results. The first is what column density to report. Since we typically not only do not know the state of the matter or indeed the precise elements we are observing, a column density of any given species cannot be reported. For this reason an equivalent column density in hydrogen is usually reported assuming, typically, a 'solar' abundance of the elements - usually the default abundance set in the software Xspec, from Anders \& Grevesse (1989). In spite of newer estimates of the solar abundances that are about $40 \%$ lower in metals (e.g. Asplund et al. 2009), it seems that the Anders \& Grevesse (1989) metallicity is a better approximation than Asplund et al. (2009) for a typical Galactic sightline (Watson 2011). For this reason and for ease of comparison with previous estimates, it makes sense to continue to use Anders \& Grevesse (1989) metallicities unless we know what the absorber is. The second complication is that the ab- 
sorbing column density is occasionally reported as if the absorber was at $z=0$, typically where the redshift is unknown. The absence of spectral features means that the redshift must be determined from observations at another wavelength, typically optical or UV. Since the absorption is shifted out of the bandpass with redshift, the observed column density drops with redshift approximately as $(1+z)^{-2.6}$. Naturally the column density reported at $z=0$ is thus substantially smaller than the actual value determined at the correct redshift. Third, the value found for the column density is almost always determined assuming a neutral column density. Ionised gas has a smaller absorbing cross-section, and indeed, a different absorbing pattern.

\section{HIGH X-RAY ABSORPTION AT HIGH REDSHIFT: WHY DOES $N_{\mathrm{H}_{\mathrm{X}}} / A_{V}$ EVOLVE WITH REDSHIFT?}

It was noted by Campana et al. (2010) that there was a correlation between the X-ray absorbing column densities in GRB afterglows and their redshift, i.e. the highest redshift bursts were the most absorbed. Behar et al. (2011) demonstrated convincingly that this was not an artifact of the fitting and assumptions, and suggested that this relation was due to increasing foreground absorption by the highlyionised metals component of the intergalactic medium. However, Watson \& Jakobsson (2012) found the ratio of the Xray absorption to dust extinction $N_{\mathrm{H}_{\mathrm{X}}} / A_{V}$ in GRB afterglows evolved with redshift, and that it was this evolution that answered the mystery of the dearth of high X-ray absorbed GRBs at low redshift. This answer was in many ways more mysterious than the question. Why would the X-raydetermined metal column density increase relative to the dust column density with redshift? Here we examine the relationship between $N_{\mathrm{H}_{\mathrm{X}}}$ and $A_{V}$ as a function of redshift to understand the origin of this peculiar evolution using (a slightly enlarged version of) the data in Watson \& Jakobsson (2012). These data are presented in detail in Watson et al. (in prep.).

A close examination of Fig. 3 in Watson \& Jakobsson (2012) hints at a possible correlation between the metal column densities and the extinction that evolved with redshift. While there is no immediate correlation apparent in the full dataset, splitting the data by redshift clarifies the situation. In Fig. 2, the distribution of X-ray absorbing column density with extinction is plotted for GRBs in four redshift intervals. The correlation then becomes more apparent, with correlation probabilities of greater than $99.9 \%, 97.5 \%$, and $96 \%$ for GRBs in the intervals $z<1,1<z<2$, and $2<z<4$ respectively based on Kendall's $\tau$. The combined probability that such correlations occur randomly is less than $1 \times 10^{-5}$. Similar probabilities are obtained using Spearman's $\rho$. While the correlation is highly significant, there is clearly significant scatter in this correlation. In sum: the X-ray absorption is correlated with the dust extinction and the relation evolves with redshift.

\subsection{Origin of the correlation between $N_{\mathrm{H}_{\mathrm{X}}}$ and $A_{V}$}

At first glance, the fact that dark bursts are preferentially associated with high $N_{\mathrm{H}_{\mathrm{x}}}$ GRBs (Krühler et al. 2011; Campana et al. 2012; Fynbo et al. 2009) implies an $N_{\mathrm{H}_{\mathrm{X}}}-A_{V}$ correlation. The evolution with redshift and the scatter however, was concealing it (e.g. Campana et al. 2010). The first interpretation might be that this is simply host ISM in both dust and metals, resulting in high $N_{\mathrm{H}_{\mathrm{X}}}$ and $A_{V}$ values. However, we know that the absorption in the optical-UV is dominated by low-ionisation gas and dust at distances of hun- dreds of pc (see Watson et al. 2007; Vreeswiik et al. 2007; Fox et al. 2008; D'Elia et al. 2009a; Campana et al. 2010; Schady et al. 2011; Vreeswijk et al. 2013). We also know that these low-ionisation metal column densities correlate well with the observed $A_{V}$ (Zafar et al. in prep., see also de Ugarte Postigo et al. 2012). For the observed $N_{\mathrm{H}_{\mathrm{X}}}-A_{V}$ correlation to hold, the cool, ISM would have to dominate the column density. And indeed, it has been argued (Krühler et al. 2011) that even with both components entirely uncorrelated, an approximate correlation between $\mathrm{H}$ I and $N_{\mathrm{H}_{\mathrm{X}}}$ would be found at high column densities if the $\mathrm{H}$ I column is sufficiently dominant.

However this cannot be the origin of the correlations we observe with the X-ray column. First, the H I column density, corrected for metallicity, is almost never as large as the observed X-ray column density (see Fig. 3, also Zafar et al. 2011; Krühler et al. 2011), and the correlation extends to low column densities. We show in Fig. 2 the X-ray absorption values after subtraction of $2.0 \times 10^{21} \mathrm{~cm}^{-2} \times A_{V}$ (Watson 2011), which is the absorption expected for the metals typically associated with such a column of dust (Zafar et al. in prep.). The remaining X-ray absorption is still correlated with $A_{V}$, with probabilities of $96 \%, 94 \%$ and $92 \%$ for the three lower redshift sets. The combined probability of these datasets all having such strong correlations by random chance is only $\sim 3 \times 10^{-4}$

Second, the correlation between the hydrogen column density and the X-ray absorbing column density is tighter before accounting for metallicity (see Figs. 3 and 4 in Campana et al. 2010). This shows that the correlation of the X-ray absorbing column density is primarily with the full gas column density and not particularly with the neutral metals. Third, there is no obvious reason there should be an evolution in the $N_{\mathrm{H}_{\mathrm{X}}} / A_{V}$ ratio with redshift in this scenario. We conclude, therefore that the typical host galaxy ISM is not responsible for the correlation and that there is a correlation between the dominant component of the X-ray absorbing column density and the total gas column density.

\subsection{High $\mathrm{X}$-ray column density, high gas column density}

Such a correlation is odd at first, since the X-ray absorption is usually principally due to metals. However, what it is telling us, is not that the gas column is responsible for absorbing the $\mathrm{X}$-rays, but that where the X-ray column density is high, so too, often, is the gas column density. This correlation with the gas column density then explains the strong redshift evolution of the $N_{\mathrm{H}_{\mathrm{X}}}-A_{V}$ relation, but the apparent lack of redshift evolution of the $N_{\mathrm{HX}}-N_{\mathrm{HI}}$ relation (Fig. 3). It is simply the average metallicity evolving with redshift. At high redshifts we have high X-ray column density objects with high gas column densities. These objects are significantly metal poorer than at low redshift, and hence (via the virtually constant dust-to-metals ratio demonstrated in Zafar et al. in prep.), have a lower $A_{V}$. In other words, the evolving $N_{\mathrm{H}_{\mathrm{X}}}-A_{V}$ relation is a reflection of the cosmic metallicity evolution.

We can now draw a few important conclusions from this. First, the X-ray absorption component must be associated with the host galaxy since it correlates with the gas column density in the host. Thus it cannot be due to the intergalactic medium, for example. Second, since the maximum values of $N_{\mathrm{H}_{\mathrm{X}}}$ are very similar at high and low redshift, and the mean distribution certainly does not decrease to high redshift, as would be expected if we were observing an X-ray absorbing 

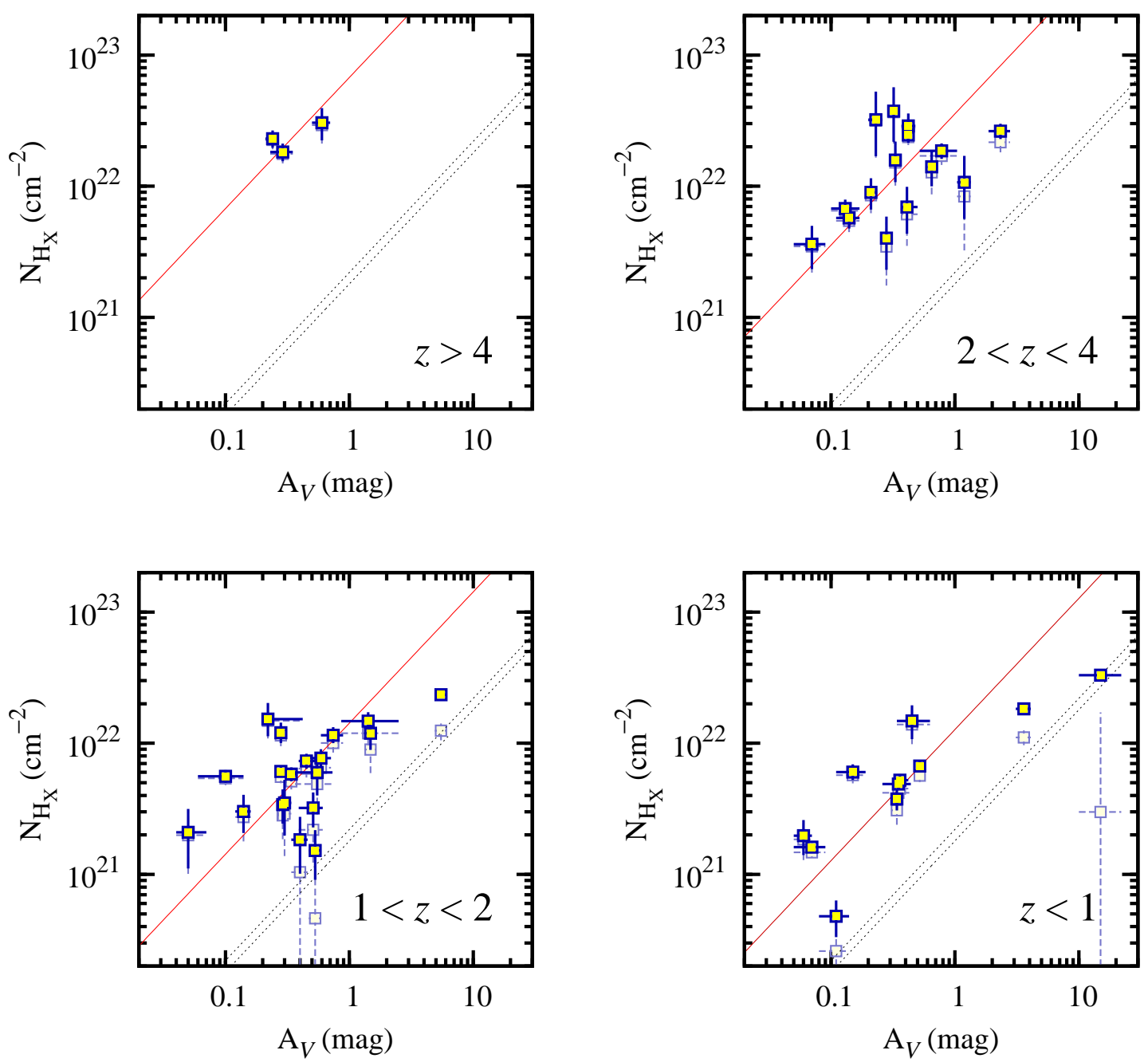

Figure 2. X-ray absorption versus extinction in GRB afterglows. The four panels present GRBs in different redshift intervals: top left $z>4$, top right $2<z<4$, bottom left $1<z<2$, bottom right $z<1$. The solid line is the best-fit, fixed-slope line to the data. The dashed lines mark the approximate limits of the metals-to-dust ratios reported for the local group of galaxies. The metal and dust columns are clearly correlated in each redshift interval $(P>95 \%$ in the three $z<4$ datasets). The best-fit metals-to-dust ratio rises at high redshift: from $\sim 1 \times 10^{22} \mathrm{~cm}^{-2} \mathrm{mag}^{-1} A_{V}$ at the lowest redshift to $\sim 6 \times 10^{22} \mathrm{~cm}^{-2} \mathrm{mag}^{-1} A_{V}$ at the highest. The datapoints in faded colours are $N_{\mathrm{H}_{\mathrm{X}}}-\left(2.0 \times 10^{21} \mathrm{~cm}^{-2} \times A_{V}\right)$ versus $A_{V}$. The remaining $\mathrm{X}$-ray absorption when the metal absorption corresponding to the near-universal metals-to-dust ratio (Zafar et al., in prep.) is subtracted $\left(2.0 \times 10^{21} \mathrm{~cm}^{-2} \times A_{V}\right)$ is still clearly correlated with $A_{V}$, showing that the $N_{\mathrm{H}_{\mathrm{X}}}-A_{V}$ correlation we observe is not due to the neutral medium metals associated with the dust forcing a correlation at high $A_{V}$ values.

column dominated by metals in the ISM gas, it suggests that we are either observing metals ejected by the progenitor star itself or that a component of the gas largely unaffected by cosmic metallicity evolution is the absorber.

\subsection{The explanation of the 'dark burst'- $N_{\mathrm{H}_{\mathrm{X}}}$ connection}

The overall correlation between the X-ray column density and the dust extinction can be explained by a model where GRB progenitors residing in the hearts of galaxies are surrounded by higher density ISM. Both H II regions and WRnebulae are known to expand to smaller sizes in the hearts of galaxies than those on the outskirts or outside the plane (Hunt \& Hirashita 2009; Stock \& Barlow 2010). These more compact sizes result in higher observed column densities through the nebula or $\mathrm{H}$ II region. GRBs occurring in high ISM density regions will therefore have higher X-ray column densities. Naturally, the afterglows of such bursts are also much more likely to encounter high density ISM sightlines on their way out of their hosts. The approximate correlation observed between the X-ray column density and the dust extinc- tion Fig. 2 is then due to this effect. The fact that 'dark bursts' have on average higher X-ray column densities (Krühler et al. 2011; Campana et al. 2012; Fynbo et al. 2009; Krühler et al. $2012 b$ ) is clearly an observational corollary.

We can confirm this explanation if we observe an overall and rough correlation between the X-ray absorbing column density and the neutral hydrogen column density. This is the case. In Fig. 3 we plot the X-ray absorption against the neutral hydrogen column density determined from the Ly $\alpha$ line. There is an overall correlation between the two (96\% confidence including all the data), with a few strongly discrepant outlying objects with very low H I column densities-which in this scenario are objects that happen to find a clean line of sight out of the host. In an earlier, smaller sample, we found no evidence for a correlation between the X-ray and H Ly $\alpha$ column densities, partly due to these few very low H Ly $\alpha$ column density objects (Watson et al. 2007).

The mysterious drop in the observed dust column for higher redshift sources (see Watson \& Jakobsson 2012) is readily explained in this context. If we assume that the average metallic- 


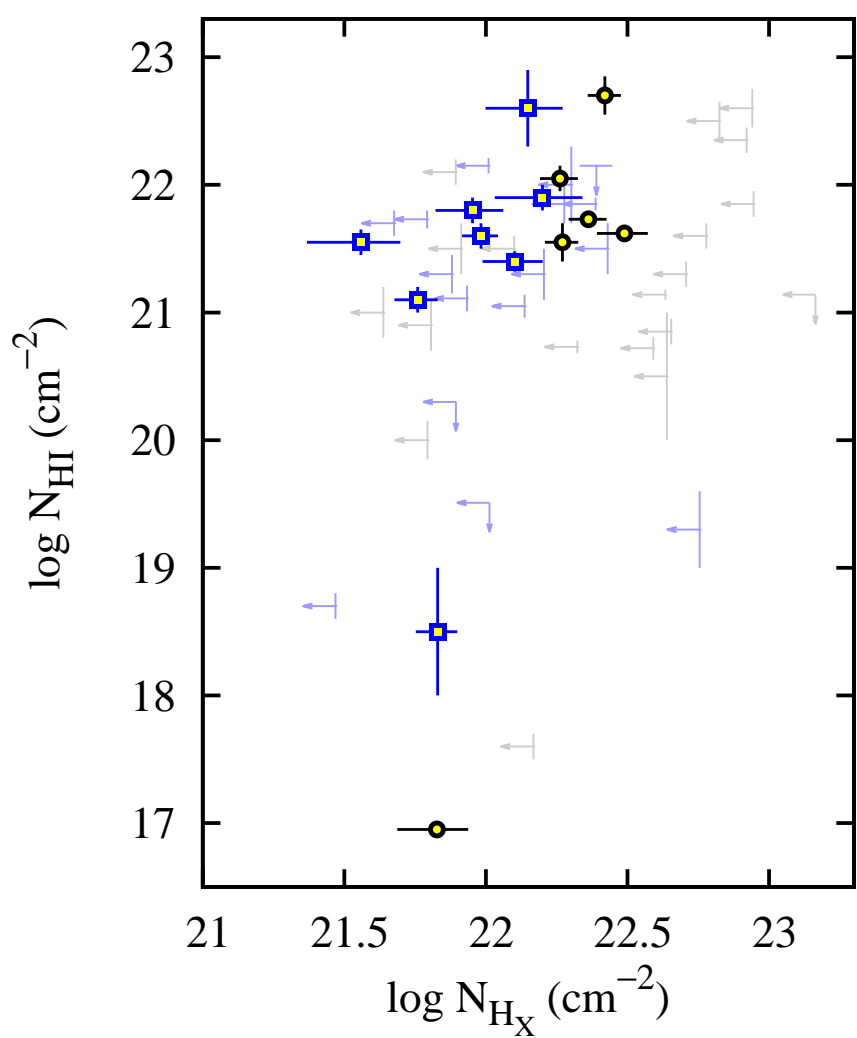

Figure 3. Gas (H I) column density as a function of X-ray absorbing column density. Objects at high $(z \geq 3)$ and low $(z<3)$ redshifts are marked with squares (blue) and circles (black) respectively. At the higher values of $N_{\mathrm{H}_{\mathrm{I}}}$, i.e. for most objects, the gas and X-ray column densities are correlated, though with a considerable intrinsic scatter. The correlation is similar to that observed between the dust extinction and the X-ray absorption (Fig. 2). However, unlike the dust correlation, there is no obvious evolution with redshift. Note that due to the low energy cut-off, the detectability threshold for the $\mathrm{X}$-ray absorption is strongly redshift dependent. This detectability effect is clear with the high redshift objects (black) having higher X-ray absorption and upper limits.

ity of galaxies is low at high redshifts and the gas columns are approximately similar for similar X-ray absorptions at all redshifts, and the dust-to-metals ratio is roughly constant, then the apparent increase in the $N_{\mathrm{H}_{\mathrm{X}}} / A_{V}$ is simply a result of the decreasing metallicities at these high redshifts. If this explanation is correct, we should observe an evolution of increasing $N_{\mathrm{H}_{\mathrm{X}}} / A_{V}$ with redshift, but no such evolution in the $N_{\mathrm{HI}} / N_{\mathrm{H}_{\mathrm{X}}}$ ratio. Again, this is what we observe, with no apparent evolution of the $N_{\mathrm{HI}} / N_{\mathrm{H}_{\mathrm{X}}}$ ratio (Fig. 3].

A potentially fascinating tool resulting from this discovery is that the observed evolution of the mean $A_{V} / N_{\mathrm{H}_{\mathrm{X}}}$ for GRBs can therefore yield an approximate indication of metalenrichment of star-forming galaxies with time. At first glance, for example, we can argue that the metallicity in star-forming galaxies decreases by approximately a factor of 3 between $z<$ 1 and $2<z<4$ and by a factor of 5 between $z<1$ and $z>4$. This is a flatter slope to the metallicity evolution than found with QSO-DLAs (Prochaska et al. 2003). GRB host metallicities, on the other hand, where they have been measured, also show an evolution with redshift, but the evolution is significantly slower than for QSO-DLAs and is quite consistent with what we observe here (Fynbo et al. 2006; Levesque et al. 2010; de Ugarte Postigo et al. 2012; Thöne et al. 2012). This is reassuring for the interpretation presented above.

\section{WHAT IS THE X-RAY ABSORBER?}

Given the correlation with gas in the host galaxy, it is clear that the X-ray absorber must have some connection to the host ISM, but not the typical free, low-ionisation ISM observed far from the burst. We are thus left with two possibilities: the immediate cloud in which the burst progenitor was born, or circumstellar material.

\subsection{The host molecular cloud}

It has been proposed that the X-ray absorption is related to gas and dust in the molecular cloud in which the GRB progenitor is formed, but that the GRB ionises the gas and destroys the dust to potentially large distances (Waxman \& Draine 2000; Fruchter et al. 2001; Galama \& Wijers 2001; Perna et al. 2003), leaving the Xray absorption by highly-ionised metals as the only trace. However two pieces of evidence argue against this possibility. First, the low-ionisation optical absorber is observed to lie at very large distances in almost every case, hardly ever at distances of a few pc (Vreeswiik et al. 2007; D'Elia et al. 2009a b, 2011; Ledoux et al. 2009; Fox et al. 2008). Molecular hydrogen is very rarely detected (Ledoux et al. 2009; Prochaska et al. 2009; Whalen et al. 2008), though there is a strong dust-extinction bias against such detections. And there is no one-to-one correlation between the X-ray absorption and the neutral hydrogen column density (Watson et al. 2007). This means that the hydrogen related to the X-ray absorber must be dissociated and ionised. However there are not enough UV continuum photons in most GRBs to dissociate and then ionise the hydrogen out to distances much greater than $\sim 1$ pc from the burst for typical inferred column densities (Waxman \& Draine 2000; Watson et al. 2007). Second, there is little evidence for the moderately ionised species one might expect to exist, lying in the zone between the highly-ionised X-ray absorber and the almost neutral optical absorber (Schady et al. 2011). Ultimately, the GRB is not UV-luminous enough to ionise the hydrogen to super-pc distances, while the molecular clouds in which massive stars form should often be larger than this size, and we do not observe any of the signatures of such molecular clouds, of their ionisation by the GRB, or of the remnant neutral/molecular material we would expect if the GRB were 'burning' its way out of its molecular cloud (see Waxman \& Draine 2000). We therefore conclude that host molecular clouds cannot be the origin of the X-ray absorption in the general case.

\subsection{The host H II region}

We must be careful to distinguish between a molecular cloud and what once was a molecular cloud, which, after only a few million years will be substantially ionised by the massive stars formed in it, transforming it into a $\mathrm{H}$ II region, likely before the burst occurs. We are then discussing H II regions rather than molecular clouds, and the arguments related to ionisation of hydrogen by the GRB do not apply. The total metal column densities are in the right range to explain the observed X-ray absorption, in H II regions with sizes up to several tens of pc in size (Fig. 4, Hunt \& Hirashita 2009). However, the principle objection to this scenario is the observation that the $N_{\mathrm{H}_{\mathrm{X}}} / A_{V}$ ratio evolves with redshift, which cannot readily be explained in this scenario unless the absorption is not due to the metals in the gas.

The absence of an observed decrease in the X-ray column density to high redshift is a strong indication that the X-ray absorber is not related to the metals in the general ISM of 
the host galaxy. If it was, the overall evolution of the cosmic metallicity would imply a decrease of two orders of magnitude in the absorption to $z=8$. If the X-ray absorption was influenced by the mean ISM metallicity, we would anticipate a strong decrease in the mean X-ray absorption. If anything, the mean X-ray absorption appears to increase with redshift (Campana et al. 2012; Watson \& Jakobsson 2012) and this cannot be a selection bias, since the total number of objects at high redshift would become unfeasibly large if there was a missing population of low-absorption systems at high redshift. In addition, there should be no strong bias against discovering (not measuring) low-absorption systems at high redshift. We cannot see how an absorber dominated by the mean metallicity of the host galaxy can reproduce the observed high absorption systems at high redshift, and therefore are compelled to exclude metals in the host H II region as the origin of the X-ray absorption. However, it is worth considering whether the $\mathrm{He}$ in the host $\mathrm{H}$ II region could provide enough $\mathrm{X}$-ray absorption. In that case we would need to demonstrate that $\mathrm{H}$ II regions have the correct distribution of gas and dust column densities, and radii, and we would have to explain the lack of observations of moderately-ionised species.

\subsection{Ejecta from the progenitor}

It is natural enough, considering the association of GRBs with type Ic SNe (Galama et al. 1998; Stanek et al. 2003; Hjorth et al. 2003), to suggest that GRBs have heavily masslosing stars (specifically, Wolf-Rayet (WR) stars) as progenitors (e.g. Wooslev 1993; Chevalier \& Li 1999). Recent observations of WR stars in the Galaxy and Magellanic clouds show that the WR nebula, filled with the highly-ionised, metal-rich ejecta of the star, has a very high X-ray column density, typically about a few $\times 10^{22} \mathrm{~cm}^{-2}$ (Koyama et al. 1994; Skinner et al. 2010; Zhekov et al. 2011; Gosset et al. 2011; Oskinova et al. 2012), that is dust-free (or at least very dust-poor compared to the typical ISM). The typical sizes of WR nebulae (a few pc) are consistent with the maximum sizes estimated for the ionised GRB absorber (Watson et al. 2007; Schady et al. 2011, though under a slightly different set of assumptions). The wind is already quite hot before the burst and so the bulk of the absorbing material is already significantly ionised and may be far enough away from the star that we would rarely if ever expect to see substantial transient ionisation by the GRB itself.

We need not confine our considerations to mass-loss scenarios as observed for known, well-observed Galactic WR stars. It appears from recent work that many evolved massive stars suffer significant mass loss in outbursts, the archetype of which is $\eta$ Carinae, where the mass loss from the $1840 \mathrm{~s}$ event has been estimated to have been as much as $15 \mathrm{M}_{\odot}$ (Smith et al. 2003). These outbursts come, presumably, from stars transitioning via precisely this mass-loss mechanism to the WR phase (Smith \& Owocki 2006). The nebulae around these high-mass stars are often dusty and very massive, and appear to be more common than previously believed (Wachter et al. 2010; Gvaramadze et al. 2010). Furthermore, such extreme mass loss episodes have been discovered in scattered light, dust reheating and absorption observed via supernovae, suggesting that eruptive mass loss is common in the last millenium right up to explosion of the star (Fox et al. 2010; Pastorello et al. 2007; Smith et al. 2011; Chandra et al. 2012).

The progenitor ejecta scenario explains many of the available observations, including the high level of the X-ray ab- sorbing column density compared to $N_{\mathrm{HI}}$ and its relative lack of evolution with redshift.

However, in spite of the case made for progenitor ejecta, a major problem remains with this interpretation: for distances of the absorber from the progenitor larger than $\sim 1 \mathrm{pc}$, the mass required becomes very large, however the luminosity of the prompt GRB and early afterglow strips light metals entirely of their electrons at anything except large distances.

This minimum distance is so large that for a given column density, the total mass required is excessive for a progenitor wind. Detailed analyses (Perna \& Lazzati 2002) as well as our own simple photon number calculations, indicate that the GRB emission strips light metals to distances of several tens of $\mathrm{pc}$ in some cases and a few pc even for lower luminosity bursts. But at distances of $1 \mathrm{pc}$, as we note above, the mass required to produce the mean observed GRB X-ray absorption is more than $1 M_{\odot}$ in metals for spherically distributed ejecta. This value rises as the square of the distance, so that at a mean distance of even a few pc, we would typically require approximately ten solar masses of light metals.

These large masses essentially mean $r \lesssim 1$ pc for progenitor ejecta, while in direct contradiction, the power of a typical burst completely ionises metals that lie closer than a few pc, thus excluding an effective absorber at distance less than a few pc (e.g. Perna \& Lazzati 2002). Highly asymmetric mass loss, with the mass ejected along the direction of the GRB, i.e. along the rotation axis of the star, could mitigate this problem, but not enough and we still need the mass to lie at distances typically smaller than $\sim 1 \mathrm{pc}$.

\section{PROPERTIES OF THE ABSORBER}

We look at the properties listed above and examine specifically the compatibility of these models with 1) the high level and distribution of $N_{\mathrm{H}_{\mathrm{X}}}, 2$ ) the evolving nature of the dust-to$N_{\mathrm{H}_{\mathrm{X}}}$ ratio, 3) the correlation between X-ray column density and the gas, 4) the large typical distances to neutral gas, 5) the absence of moderately-ionised species, and 6) any reports of features related to metals in the X-ray absorber.

\subsection{H II region or progenitor nebula?}

Both the progenitor ejecta and natal H II region work well to reproduce the observed features of the X-ray absorption. In particular, the correlation between the X-ray absorbing column density and the gas column density strongly suggests that the magnitude of the X-ray column is driven largely by confinement by the host ISM, i.e. for a given mass, the column density through it decreases as the square of the radius. As observed for WR nebulae and nearby H II regions, the radius of the region is related to galacto-centric distance (Hunt \& Hirashita 2009), e.g. by far the largest WR ejecta nebula is above the Galactic plane (W71, Stock \& Barlow 2010). For a given ejected mass, a physically smaller bubble will result in a higher column density, giving rise to the relation we observe in Figs. 2 and 3 .

However both hypotheses suffer from difficulties. In the former scenario there is a 'mass-distance problem': a tension between the minimum distance the metals must lie at in order not to be stripped of all their electrons by the GRB and the maximum distance allowable to keep the mass required to reproduce the observed column densities at a reasonable level. We explore possible solutions to the mass-distance problem in the appendix, but find no solution consistent with the observed data and therefore reject it. In the latter scenario, the X-ray absorbing column density should drop with redshift as the mean 
metallicity of star-forming regions drop. A He-dominated H II region absorption would resolve this issue. We explore this scenario below.

\subsection{Properties of a helium-dominated H II region absorber}

We examine here more quantitatively the prospect that the host $\mathrm{H}$ II region could have the properties required to reproduce the X-ray absorption.

The reason $\mathrm{He}$ comes to dominate the X-ray spectra of GRB afterglows is because the stripping radii related to a GRB are, somewhat counterintuitively, smallest for $\mathrm{H}$, then $\mathrm{He}$ and then the metals considerably further out. This is because the GRB emission has a far harder spectrum than even hot stars; in photon flux terms, $F(E) \propto E^{-\Gamma}$, where $\langle\Gamma\rangle \simeq$ 1 (Kaneko et al. 2006). The number density of the atoms thus dominates the ionisation distances, and so those elements with higher number densities have the smaller stripping radii. Therefore we would expect $\mathrm{H}$ and $\mathrm{He}$ to feature prominently in the afterglow absorption, especially in low metallicity environments where GRBs are found (Modjaz et al. 2008; Graham \& Fruchter 2012). However, in H II regions, the hydrogen is of course pre-ionised by the stars, while the $54 \mathrm{eV}$ photons required to strip $\mathrm{He}$ are extremely rare, making $\mathrm{He}$ the dominant absorber. Indeed, we would expect a priori that He should absorb GRB afterglows very strongly and it's worth asking the question: if anything else were responsible for the excess X-ray absorption, then why don't we see He absorption?

We can calculate very roughly the effective stripping radius of various elements making somewhat simplistic assumptions equating the total flux of available photons and the total crosssections of the hydrogen- and helium-like ions (i.e. with only one or two electrons remaining) at these radii. We have done this for a representative, well-studied GRB, GRB 050401 at $z=2.9$ (Watson et al. 2006) and show these values in Fig. 4 It is clear that the space where the ionisation state of He remains largely unaffected is very significant compared to the stripping radius for $\mathrm{O}$ (the stripping radius for $\mathrm{Fe}$ is similar to $\mathrm{O}$, and is further out for $\mathrm{C}$ and other metals), leaving $\mathrm{He}$ as the only absorber in the region between about $5 \mathrm{pc}$ and $\sim 30 \mathrm{pc}$. This marks the effective size of the $\mathrm{H}$ II region surrounding GRB 050401. It must be larger than $5 \mathrm{pc}$ for there to be any He to absorb, and must be smaller than $\sim 30 \mathrm{pc}$, so that the metals and dust do not start to contribute substantially to the optical/UV spectrum. This limit on the size and our measurment of the column density also allows us to determine that the density of the $\mathrm{H}$ II region must be in the range $10^{3}-10^{4} \mathrm{~cm}^{-3}$. These numbers are consistent with the densities found for nearby H II regions, though we should note that GRB 050401 was a very luminous burst. In Fig. 4 we plot the effective X-ray column density expected from $\mathrm{He}$ II for the densities and sizes of $\mathrm{H}$ II regions in the sample presented by Hunt \& Hirashita (2009). The mean columns for the radio-determined densities and sizes are surprisingly close the mean columns found for GRB afterglows $\left(\log N_{\mathrm{H}_{\mathrm{x}}} \sim 21.7\right)$. The column densities determined from the HST observations are consistently lower and may be affected by the resolution of the HST imaging and ground-based spectroscopy (Hunt \& Hirashita 2009).

We have also plotted the thermal sublimation radius for dust according to the prescription of Waxman \& Draine (2000). However, we note that in that paper they assumed only photons in the range $1-7.5 \mathrm{eV}$ would contribute to the dust heat-

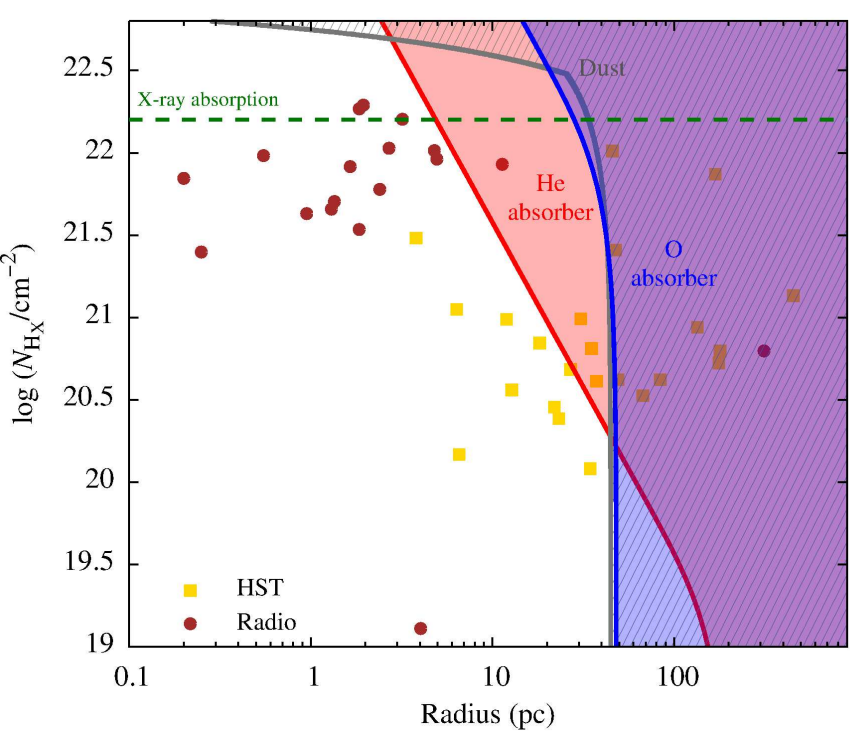

Figure 4. Equivalent hydrogen column density $\left(N_{\mathrm{H}_{\mathrm{X}}}\right)$ as a function of radius for a sample of nearby extragalactic star-forming regions from Hunt \& Hirashita (2009) including many blue compact dwarf galaxies derived from radio observations (circles) and HST imaging with ground-based spectroscopy (squares). The distribution of the radio-derived column densities, while containing possibly severe selection biases, is compatible with the mean observed $N_{\mathrm{H}_{\mathrm{X}}}$ in GRBs (Watson \& Jakobsson 2012; Campana et al. 2012), indicating that the bulk of the X-ray absorption in GRBs could arise from material in its surrounding $\mathrm{H}$ II region. The column density for GRB 050401 is indicated by a dashed line. The white region indicates the distance to which GRB 050401 has enough soft X-ray photons to fully strip helium and metals so that no X-ray absorption would be observed from the $\mathrm{H}$ II region with a radius smaller than this distance in an afterglow. Helium is stripped to smaller distances than oxygen (or other metals). Dust will be destroyed by thermal sublimation to large radii. The survival distances of $\mathrm{He}, \mathrm{O}$ and dust from GRB 050401 are indicated by red, blue and shaded regions respectively. There is a large region where only He retains its electrons, making it the likely dominant X-ray absorber for most GRBs.

ing since molecular and atomic hydrogen would absorb the UV photons above this energy. We use the parameters for the GRB 050401 burst from the Swift-BAT automated analysis, as well as all the photons up to $40 \mathrm{eV}$, since there is no $\mathrm{H}_{2}$ or H I. This more than doubles the effective thermal dust sublimation radius. The dust sublimation radius is rather close to the $\mathrm{O}$ and $\mathrm{Fe}$ stripping radii, and dust is therefore unlikely to play a role in the absorption by the H II region, again, leaving only $\mathrm{He}$ as the expected signature. The outcome of these considerations aligns comfortably with the fact that the optical/UV extinction observed in GRB afterglows is consistent with the column density of low-ionisation metals observed far from the burst (Zafar et al. in prep.) and with the fact that only very small column densities of moderately ionised metals are found in the spectra of GRB afterglows (Schady et al. 2011).

While $\mathrm{H} \mathrm{Ly} \alpha$ is commonly observed in the optical spectra of $z \gtrsim 1.8 \mathrm{GRB}$ afterglows, absorption lines associated with $\mathrm{He}$ are much more difficult to detect. The only lines we could reasonably expect to observe in absorption are far into the UV, at or above the He Lyman series. For singlyionised $\mathrm{He}$, this effectively means that the absorption is never observable, since for $z \lesssim 3$, the continuum photons in the He II Ly $\alpha$ region of the spectrum are absorbed by neutral hydrogen in the Galaxy, and for $z \gtrsim 3$, the continuum is absorbed by the He Ly $\alpha$ forest. The proportion of He I to He II depends of course on the hardness of the radiation field ionising the region. However, for neutral He, a space-based, UV spectro-

${ }^{6}$ http://gcn.gsfc.nasa.gov/notices_s/113120/BA/ 
graph might find the He I resonance line related to the X-ray absorpton for GRBs at $1 \lesssim z \lesssim 2.5$, dependent on the proportion of He I present.

\section{IMPLICATIONS OF HELIUM ABSORPTION}

We have concluded above that the data on X-ray absorption from GRBs allow us to exclude every scenario proposed to explain it to date with the exception of $\mathrm{He}$ in the natal $\mathrm{H}$ II region. Specifically,

a) the absence of a strong decrease in the mean X-ray absorption with redshift strongly implies that the X-ray absorber is not primarily due to metals in the ISM of the host galaxy;

b) the correlation we find between $N_{\mathrm{H}_{\mathrm{X}}}$ and $A_{V}$ implies an association between the $\mathrm{X}$-ray absorber and the host galaxy;

c) the evolution of the $N_{\mathrm{H}_{\mathrm{X}}} / A_{V}$ ratio coupled with the apparent lack of evolution of this ratio for the optical metals to dust ratio (Zafar et al. in prep.) suggests that the $\mathrm{X}$-ray absorber is not due to the metals in the dust that cause the observed $A_{V}$;

d) that the evolution of the dust column is related to the metallicity evolution, while the X-ray absorber is largely independent of this;

f) this in turn suggests that the X-ray absorber is due to $\mathrm{He}$ in the natal $\mathrm{H}$ II region or progenitor ejecta metals;

e) the ionising power of the GRB precludes metals from the progenitor ejecta;

g) the correlation between the X-ray absorbing column density and the dust extinction and the atomic hydrogen column density indicates that the host galaxy ISM has an influence on the X-ray column density.

We are thus left with a scenario in which the X-ray absorption is caused by $\mathrm{He}$ absorption in the natal $\mathrm{H}$ II region that is undetected at UV/optical wavelengths and that is confined by the host galaxy ISM. Every other scenario proposed so far is excluded.

This conclusion leads to some predictions about the observable properties of the absorber. First, as mentioned above, there might be a detectable signature of neutral He resonance absorption for GRBs in the redshift range $1 \lesssim z \lesssim 2.5$, depending on the temperature of the $\mathrm{H}$ II region. Second, since the absorption is dominated by $\mathrm{He}$, the absorption observed in X-rays should be smooth, i.e. absorption edges or lines due to metals should be weak. The claimed detection of $\mathrm{S}$ and $\mathrm{Ne}$ absorption edges in the afterglow of GRB 090618 seems problematic in this respect (Campana et al. 2011). Campana et al. (2011) found in their analysis that $\mathrm{S}$ and Ne were significantly overabundant (though without $\mathrm{Si}$ or $\mathrm{O}$ being similarly overabundant, which might have been expected on nucleosynthetic grounds), but that the general metal abundance found was low. However, it may be possible that the Galactic foreground was underestimated: an abundance low in metals (particularly in O) was assumed (Wilms et al. 2000) for the Galactic foreground absorption, substantially lower than typical for Galactic sightlines (Watson 2011). At the same time, the Ne I edge at $z=0.54$, the redshift of the GRB, is very close in energy to the Galactic O I edge. Clearly, accounting for the Galactic $\mathrm{O}$ I edge is crucial to an accurate determination of the $z=0.54 \mathrm{Ne}$ I edge. Furthermore, observationally, Campana et al. (2011) found the $\mathrm{S}$ and $\mathrm{Ne}$ abundances to be strongly correlated, such that if the $\mathrm{Ne}$ abundance was low, the $\mathrm{S}$ abundance would also likely be low. Therefore we believe that the detection of absorption edges in GRB 090618 may need to be revisited. No other non-transient absorption feature has been reported in GRB afterglows, which is perhaps noteworthy in its own right, (though we are unaware of any study explicitly searching for these features in low-redshift GRB afterglows). Currently therefore, the data appear to be consistent with a smooth X-ray absorption.

Fits performed previously to the X-ray spectra of GRB afterglows with solar metallicity material may therefore be somewhat unreliable not just in the absolute value of the derived equivalent hydrogen column density, as is generally acknowledged, but also in shape, especially at low redshift where the $\mathrm{O}$ absorption edge is especially prominent. The very low redshift GRB 060218, which is strongly absorbed, has an evolving thermal component reported (Campana et al. 2006b; Ghisellini et al. 2007). The fits to this thermal component may be significantly affected by a He-dominated absorber instead of a solar metallicity absorber.

In addition to thermal components in low redshift bursts, the conclusions drawn about the metallicity of highly absorbed bursts, especially at high redshift, based on the Xray absorption assuming the absorption to be dominated by metals, should also be revisited in light of our results (Campana et al. 2007; Watson \& Laursen 2011).

This first successful interpretation of the X-ray absorption allows it to be used for diagnostic purposes. Based on the luminosity of the burst and the continued existence of neutral or singly-ionised $\mathrm{He}$, we can set lower bounds to the radii of the $\mathrm{H}$ II region in which the burst resides, as well as upper limits based on the fact that the metals must be stripped by the burst. With such limits, we can then convert the observed column density to volume densities for these regions. Ultimately, it should be possible to calculate approximate distributions for the densities and sizes of individual H II regions where massive stars form across a vast range of redshifts, currently even as far as GRB 050904 at $z=6.3$.

\section{CONCLUSIONS}

We have shown that the X-ray absorption in GRBs is correlated with the dust extinction and with the neutral hydrogen column and that the ratio of $N_{\mathrm{H}_{\mathrm{X}}} / A_{V}$ changes with redshift in a way similar to the metallicity redshift evolution of GRB host galaxies. This suggests that the magnitude of the X-ray absorbing column density in GRBs is correlated with the gas density in the host galaxy ISM, explaining the relationship found between high $N_{\mathrm{H}_{\mathrm{X}}}$ bursts and so-called 'dark bursts'. Using these new findings, we excluded all models relating the X-ray absorber to anything outside the host galaxy including the warm-hot IGM. On the grounds that the $N_{\mathrm{H}_{\mathrm{X}}} / A_{V}$ changes with redshift, we concluded that only helium in the host $\mathrm{H}$ II region or metals ejected by the progenitor star could be the primary X-ray absorber. However, the ionising power of the GRB sets a minimum distance for metals to retain any electrons and hence be effective X-ray absorbers; this places a minimum mass on the metals required which is too large for ejecta from the progenitor, excluding this hypothesis. We thus concluded that helium in the host H II region causes most of the X-ray absorption observed in GRB afterglows. This conclusion allowed us to set limits on the sizes and densities of 
the typical $\mathrm{H}$ II regions in which X-ray absorbed GRBs explode, $\gtrsim$ a pc and $\lesssim$ a few tens of pc, with densities about $10^{3}-10^{4} \mathrm{~cm}^{-3}$, consistent with observations of sizes and electron densities of H II regions in the Milky Way and in nearby galaxies.

We would like to thank Paul Crowther, Nial Tanvir, Davide Lazzati, Enrico Ramirez-Ruiz, James Rhoads, and Peter Jakobsen for invaluable discussions. The Dark Cosmology Centre is funded by the DNRF. GL is supported by the Swedish Research Council through grant No. 623-2011-7117.

\section{REFERENCES}

Anders, E., \& Grevesse, N. 1989, Geochim. Cosmochim. Acta, 53, 197 Asplund, M., Grevesse, N., Sauval, A. J., \& Scott, P. 2009, ARA\&A, 47, 481 Behar, E., Dado, S., Dar, A., \& Laor, A. 2011, ApJ, 734, 26

Bohlin, R. C., Savage, B. D., \& Drake, J. F. 1978, ApJ, 224, 132

Bouchet, P., Lequeux, J., Maurice, E., Prevot, L., \& Prevot-Burnichon, M. L. 1985, A\&A, 149, 330

Burstein, D., \& Heiles, C. 1978, ApJ, 225, 40

Butler, N. R., \& Kocevski, D. 2007, ApJ, 663, 407

Butler, N. R., Marshall, H. L., Ricker, G. R., Vanderspek, R. K., Ford, P. G., Crew, G. B., Lamb, D. Q., \& Jernigan, J. G. 2003, ApJ, 597, 1010

Butler, N. R., Ricker, G. R., Ford, P. G., Vanderspek, R. K., Marshall, H. L., Jernigan, J. G., Garmire, G. P., \& Lamb, D. Q. 2005, ApJ, 629, 908

Campana, S., D’Avanzo, P., Lazzati, D., Covino, S., Tagliaferri, G., \& Panagia, N. 2011, MNRAS, 418, 1511

Campana, S., Thöne, C. C., de Ugarte Postigo, A., Tagliaferri, G., Moretti, A., \& Covino, S. 2010, MNRAS, 402, 2429

Campana, S., et al. 2006a, A\&A, 449, 61

-. 2006b, Nature, 442, 1008

-. 2007, ApJ, 654, L17

-. 2012, MNRAS, 421, 1697

Chandra, P., Chevalier, R. A., Chugai, N., Fransson, C., Irwin, C. M., Soderberg, A. M., Chakraborti, S., \& Immler, S. 2012, ApJ, 755, 110 Chevalier, R. A., \& Li, Z.-Y. 1999, ApJ, 520, L29

Dai, X., \& Kochanek, C. S. 2009, ApJ, 692, 677

Dai, X., Kochanek, C. S., Chartas, G., \& Mathur, S. 2006, ApJ, 637, 53

de Ugarte Postigo, A., et al. 2012, A\&A, 548, A11

D’Elia, V., Campana, S., Covino, S., D'Avanzo, P., Piranomonte, S., \& Tagliaferri, G. 2011, MNRAS, 418, 680

D'Elia, V., et al. 2009a, ApJ, 694, 332

-. 2009b, A\&A, 503, 437

Diplas, A., \& Savage, B. D. 1994, ApJ, 427, 274

Dobashi, K., Bernard, J.-P., Hughes, A., Paradis, D., Reach, W. T., \& Kawamura, A. 2008, A\&A, 484, 205

Fitzpatrick, E. L. 1985a, ApJ, 299, 219

-. 1985b, ApJS, 59, 77

-. 1986, AJ, 92, 1068

Fox, A. J., Ledoux, C., Vreeswijk, P. M., Smette, A., \& Jaunsen, A. O. 2008, A\&A, 491, 189

Fox, O. D., Chevalier, R. A., Dwek, E., Skrutskie, M. F., Sugerman,

B. E. K., \& Leisenring, J. M. 2010, ApJ, 725, 1768

Fruchter, A., Krolik, J. H., \& Rhoads, J. E. 2001, ApJ, 563, 597

Fynbo, J. P. U., et al. 2006, A\&A, 451, L47

-. 2009, ApJS, 185, 526

Galama, T. J., \& Wijers, R. A. M. J. 2001, ApJ, 549, L209

Galama, T. J., et al. 1998, Nat, 395, 670

Gendre, B., Galli, A., Corsi, A., Klotz, A., Piro, L., Stratta, G., Boër, M., \& Damerdji, Y. 2007, A\&A, 462, 565

Ghisellini, G., Ghirlanda, G., \& Tavecchio, F. 2007, MNRAS, 382, L77

Gordon, K. D., Clayton, G. C., Misselt, K. A., Landolt, A. U., \& Wolff, M. J. 2003, ApJ, 594, 279

Gorenstein, P. 1975, ApJ, 198, 95

Gosset, E., De Becker, M., Nazé, Y., Carpano, S., Rauw, G., Antokhin, I. I., Vreux, J.-M., \& Pollock, A. M. T. 2011, A\&A, 527, A66

Graham, J. F., \& Fruchter, A. S. 2012, ArXiv e-prints, 1211.7068
Güver, T., \& Özel, F. 2009, MNRAS, 400, 2050

Gvaramadze, V. V., Kniazev, A. Y., \& Fabrika, S. 2010, MNRAS, 405, 1047

Hjorth, J., et al. 2003, Nat, 423, 847

Hunt, L. K., \& Hirashita, H. 2009, A\&A, 507, 1327

Jakobsson, P., et al. 2004, A\&A, 427, 785

Kaneko, Y., Preece, R. D., Briggs, M. S., Paciesas, W. S., Meegan, C. A., \& Band, D. L. 2006, ApJS, 166, 298

Koornneef, J. 1982, A\&A, 107, 247

Koyama, K., Maeda, Y., Tsuru, T., Nagase, F., \& Skinner, S. 1994, PASJ, 46, L93

Krühler, T, et al. 2011, A\&A, 534, A108

-. 2012a, GRB Coordinates Network, 13930, 1

- 2012b, ApJ, 758, 46

Ledoux, C., Vreeswijk, P. M., Smette, A., Fox, A. J., Petitjean, P., Ellison,

S. L., Fynbo, J. P. U., \& Savaglio, S. 2009, A\&A, 506, 661

Levesque, E. M., Berger, E., Kewley, L. J., \& Bagley, M. M. 2010, AJ, 139, 694

Martin, N., Maurice, E., \& Lequeux, J. 1989, A\&A, 215, 219

Mirabal, N., Paerels, F., \& Halpern, J. P. 2003, ApJ, 587, 128

Modjaz, M., et al. 2008, AJ, 135, 1136

Oskinova, L. M., Gayley, K. G., Hamann, W.-R., Huenemoerder, D. P., Ignace, R., \& Pollock, A. M. T. 2012, ApJ, 747, L25

Pastorello, A., et al. 2007, Nature, 447, 829

Perna, R., \& Lazzati, D. 2002, ApJ, 580, 261

Perna, R., Lazzati, D., \& Fiore, F. 2003, ApJ, 585, 775

Predehl, P., \& Schmitt, J. H. M. M. 1995, A\&A, 293, 889

Prochaska, J. X., Gawiser, E., Wolfe, A. M., Castro, S., \& Djorgovski, S. G. 2003, ApJ, 595, L9

Prochaska, J. X., et al. 2009, ApJ, 691, L27

Rachford, B. L., et al. 2009, ApJS, 180, 125

Reichart, D. E., \& Price, P. A. 2002, ApJ, 565, 174

Schady, P., Savaglio, S., Krühler, T., Greiner, J., \& Rau, A. 2011, A\&A, 525, A113

Schady, P., et al. 2010, MNRAS, 401, 2773

Schlegel, D. J., Finkbeiner, D. P., \& Davis, M. 1998, ApJ, 500, 525

Shull, J. M., \& van Steenberg, M. E. 1985, ApJ, 294, 599

Skinner, S. L., Zhekov, S. A., Güdel, M., Schmutz, W., \& Sokal, K. R. 2010, AJ, 139, 825

Smith, N., Gehrz, R. D., Campbell, R., Kassis, M., Le Mignant, D., Kuluhiwa, K., \& Filippenko, A. V. 2011, MNRAS, 418, 1959

Smith, N., Gehrz, R. D., Hinz, P. M., Hoffmann, W. F., Hora, J. L.,

Mamajek, E. E., \& Meyer, M. R. 2003, AJ, 125, 1458

Smith, N., \& Owocki, S. P. 2006, ApJ, 645, L45

Stanek, K. Z., et al. 2003, ApJ, 591, L17

Stock, D. J., \& Barlow, M. J. 2010, MNRAS, 409, 1429

Tanvir, N. R., Wiersema, K., Levan, A. J., Starling, R. L. C., Margheim, S.,

\& Hayward, T. 2012, GRB Coordinates Network, 13929, 1

Thöne, C. C., et al. 2012, ArXiv e-prints, 1206.2337

Vreeswijk, P. M., et al. 2007, A\&A, 468, 83

-. 2013, A\&A, 549, A22

Wachter, S., Mauerhan, J. C., Van Dyk, S. D., Hoard, D. W., Kafka, S., \&

Morris, P. W. 2010, AJ, 139, 2330

Watson, D. 2011, A\&A, 533, A16

Watson, D., Hjorth, J., Fynbo, J. P. U., Jakobsson, P., Foley, S., Sollerman, J., \& Wijers, R. A. M. J. 2007, ApJ, 660, L101

Watson, D., \& Jakobsson, P. 2012, ApJ, 754, 89

Watson, D., \& Laursen, P. 2011, A\&A, 527, A104

Watson, D., Reeves, J. N., Osborne, J. P., Tedds, J. A., O’Brien, P. T., Tomas,

L., \& Ehle, M. 2002, A\&A, 395, L41

Watson, D., et al. 2006, ApJ, 652, 1011

Waxman, E., \& Draine, B. T. 2000, ApJ, 537, 796

Welty, D. E., Xue, R., \& Wong, T. 2012, ApJ, 745, 173

Whalen, D., Prochaska, J. X., Heger, A., \& Tumlinson, J. 2008, ApJ, 682, 1114

Wilms, J., Allen, A., \& McCray, R. 2000, ApJ, 542, 914

Woosley, S. E. 1993, ApJ, 405, 273

Zafar, T., Watson, D., Fynbo, J. P. U., Malesani, D., Jakobsson, P., \& de

Ugarte Postigo, A. 2011, A\&A, 532, A143

Zhekov, S. A., Gagné, M., \& Skinner, S. L. 2011, ApJ, 727, L17 
APPENDIX

POSSIBLE SOLUTIONS TO THE MASS-DISTANCE PROBLEM FOR PROGENITOR EJECTA

The mass-distance problem could possibly be resolved if the afterglow doesn't see the same absorption as the prompt emission, i.e. the material absorbing the afterglow is somehow not flash-stripped by the GRB. One apparently plausible scenario is that the afterglow has a substantially larger opening angle than the prompt emission. In this case, while the prompt emission may strip metals to very large radii, it does so along a very narrow beam, while the majority of the afterglow emission travels through $\mathrm{H}$ and He-like-ionised material much closer to the jet head. Another possible solution might be to clump the ejecta. Finally, we could conceal the metals in the solid phase. In this latter case, optical absorption lines would not be observed. However, we would then need to resolve the lack of observed extinction and the dust destroying power of the GRB, both of which might be resolved by placing most of the metals in large grains. We explore these possibilities below.

\section{Implications of different prompt/afterglow opening angles}

A prediction of the different opening angles of the prompt and afterglow emission is that the prompt phase should have much lower column densities on average than the afterglow. Whether this applies to later X-ray flares is unclear, as they may have the same opening angle as the jet. Some fraction of cases where Swift-XRT follow-up of a very long burst, or a burst with a precursor is rapid enough, should show absorption clearly rising as the burst transitions from the prompt to afterglow phases. So far, however, this has not been observed. Indeed, the opposite has been reported for GRB 050904 (Gendre et al. 2007; Campana et al. 2007), and in our data we do not see clear evidence for a negative difference between prompt and afterglow absorption, in direct conflict with the requirements of this hypothesis. It therefore seems unlikely that the GRB prompt and afterglow emission see very different columns of gas.

Other solutions worth considering for the mass-distance problem are that the X-ray absorber is clumped into extremely high column density knots, or extremely high volume density knots. In the former case, the knots have high enough column density that even at distances of $0.1 \mathrm{pc}$, the burst is not powerful enough to strip all the electrons. The covering fraction must be extremely close to unity, however, and for the highest column density situations, the absorber must be close to the Compton optical depth. The extremely large covering fractions seem unlikely, making this scenario interesting but low probability. In the latter case, very high volume densities could potentially allow recombination on a short timescale (e.g. $\sim 1 \mathrm{~s})$. Such high volume densities, even at $0.1 \mathrm{pc}$, imply an incredible relative thickness of the shell $\left(\sim 10^{-10}\right)$, indicating that this scenario is essentially impossible.

\section{Large grain hypothesis}

$\mathrm{X}$-rays remove the inner electrons from metals. Once this happens, the electrons do not recombine in an observable time, even for naked nuclei. This is the problem we are trying to overcome. By placing the metals in the solid phase, the affected ion can recombine rapidly, and the energy in the liberated electron can be dissipated and radiated away at longer wavelengths. This process is more effective in larger grains. The question is whether any substantial fraction of the dust can survive the intensity of the GRB at distances of a fraction of a pc, since it has been shown theoretically that the extreme radiation field of a GRB is sufficient to destroy dust to very large distances (Waxman \& Draine 2000; Fruchter et al. 2001; Perna \& Lazzati 2002). We hypothesise that the metals responsible for the X-ray absorption could be primarily in large $(\sim 1 \mu \mathrm{m})$ dust grains. First, such grains are more robust to UV sublimation. The UV heating is approximately proportional to the surface area of the grain. Larger grains have a higher volume to surface ratio and are thus more robust to sublimation. Second, such large grains could solve the problem that we do not observe substantial reddening associated with the X-ray absorber (Perna et al. 2003). However, on closer inspection, it appears they cannot. The absorption in X-rays has the characteristic spectral shape of neutral medium absorption. Specifically, the spectrum is not flat at low energies, indicating the covering fraction is close to unity. This means that if metals in large grains are responsible for the X-ray absorption, the grains must have a covering fraction close to unity. For most dust materials, the absorption efficiency is close to unity. Even for highly-transparent materials with relatively low absorption in the optical (e.g. diamond), and large grains, the scattering efficiency is close to unity, which would lead to very strong extinction of the optical and UV light. We thus conclude that we cannot find a way to reconcile the progenitor ejecta hypothesis with the observed properties of GRB afterglows. 